\title{
The concentration of energy from Edge Lasers by a Diffractive Optical Component
}

\author{
A. Sobczyk, J. Suszek, A. Kołodziejczyk, and M. Sypek \\ Faculty of Physics, Warsaw University of Technology, Koszykowa 75, 00-662 Warszawa,
}

Received October 23, 2016; accepted November 03, 2016; published December 31, 2016

\begin{abstract}
The aim of this paper is to design a diffractive optical element based on a laser junction numerical model. We assumed a "long" laser junction emitting quasi-monochromatic, spatially incoherent light that corresponds to a high power laser. A diffractive optics approach can be used due to narrowband illumination. An especially designed numerical junction model was experimentally verified. Then a numerically designed diffractive focusing element was manufactured and tested in the experimental setup. This paper includes theoretical investigations, numerical modeling as well as experimental data.
\end{abstract}

High power edge emitting laser diodes have an active area which is relatively narrow in one direction $(y$, about $1 \mu \mathrm{m})$ and much wider in the perpendicular direction $(x$, an order of $100 \mu \mathrm{m})$. This results in a number of modes being generated along the junction leading to reduced spatial coherence [1-2].

The distribution of the radiation pattern of laser diodes (LDs) was investigated in many papers [3-7]. A valuable theoretical model based on the shifted elementary field method [8-9] was proposed by Partanen et al. [10]. In our work we make a similar assumption, i.e. the laser junction is composed of elementary fully coherent sources which are not mutually correlated. We perform the modelling of the active area of the laser diode in such a way that nonparaxial propagation [11-14] leads us to measured far field distribution. Finally, we use such an approach to design the diffractive structure shaping the beam coming from the LD in the expected way.

Our model is based on the assumption that the laser junction is divided into coherent sources being mutually incoherent. Therefore, the intensity of light at a given distance behind the junction can be considered as a sum of intensities coming from particular coherent segments. By choosing proper parameters one can obtain a theoretical model of the junction which allows to numerically simulate the light field distribution measured in the experiment after the propagation along the $\mathrm{z}$ distance. The amplitude of the light field within the LD active area in the $y$ direction was thoroughly described in the literature.

*E-mail: artur.sobczyk@if.pw.edu.pl
Following Ref. [5], we assume that the amplitude distribution in the $y$ direction is $u_{0} \exp [-p|y|]$, where $u_{\mathrm{o}}$ and $p$ are constants. The amplitude in the $x$ direction can be described as the Gaussian modes distribution [5]. Therefore, our model assumes that the output plane of the LD consists of elementary coherent sources of constant phases and amplitudes described in the $x$ direction by the Gaussian function.

One can obtain the numerical model of the laser junction by the incoherent summation of intensities of coherent sources. Therefore, the intensity distribution of the laser diode light field in the junction plane can be written as follows:

$$
I(x, y)=\left|u_{0}\right|^{2} \sum_{i=1}^{N}\left|\exp \left(-\frac{\left(x-\frac{a \cdot i}{N}\right)^{2}}{2 \cdot \sigma^{2}}\right) \exp (-p|y|)\right|^{2},
$$

Where $u_{\mathrm{o}}$ is constant, $a$ denotes the length of the junction, $N$ is the number of elementary sources within the junction and $\sigma$ describes the profile of the Gaussian curve.

In order to achieve a good model of field distribution in the LD output plane, one should find proper values of $N, \sigma, p$. The $p$ parameter corresponds to the light amplitude within the LD active area in the $y$ direction and we assume that it can be found independently from $N$ and $\sigma$. By changing $N$ (the number of coherent elementary sources) one should find $\sigma$ defining the sufficiently homogeneous amplitude of the laser active area in the $x$ direction. All parameters are changed until the far field intensity distribution corresponds closely to the measured one.

The PerkinElmer PGAS1S06H laser diode model was designed and investigated. The emitting wavelength was determined as $904 \mathrm{~nm}$.

The numerical calculations were based on the modified convolution method for a non-paraxial approach [11-14]. All calculations were carried out for the wavelength $\lambda=0.904 \mu \mathrm{m}$ on the matrix $16384 \times 16384$ points with the sampling $0.25 \mu \mathrm{m} \times 0.25 \mu \mathrm{m}$. The distance of light propagation i.e. the distance between the laser output plane and the observation plane was chosen as $1 \mathrm{~mm}$. We found that the best approximation is achieved for $a=140 \mu \mathrm{m}, N=22, p=2.02$. 
The intensity distribution measured in the plane located at a distance of $1 \mathrm{~mm}$ behind the junction is shown in Fig. 1. The theoretical curve was fitted and presented as a dotted line.

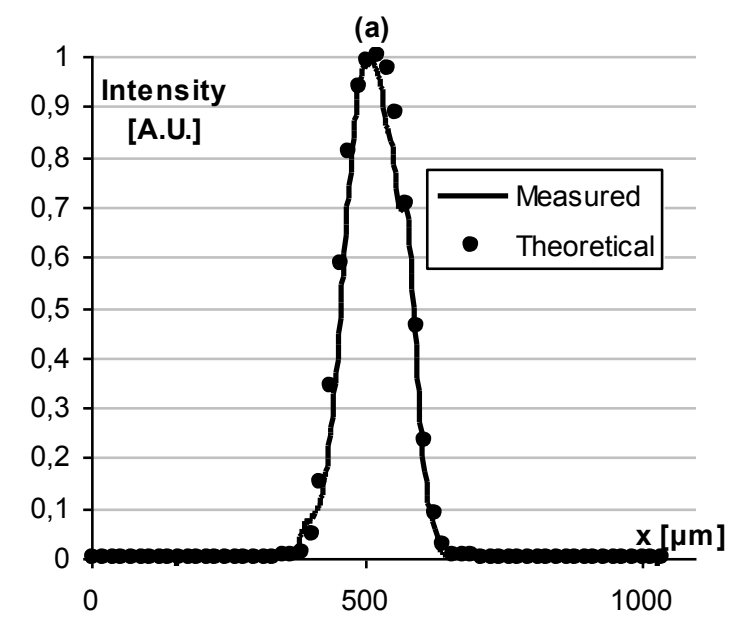

(b)

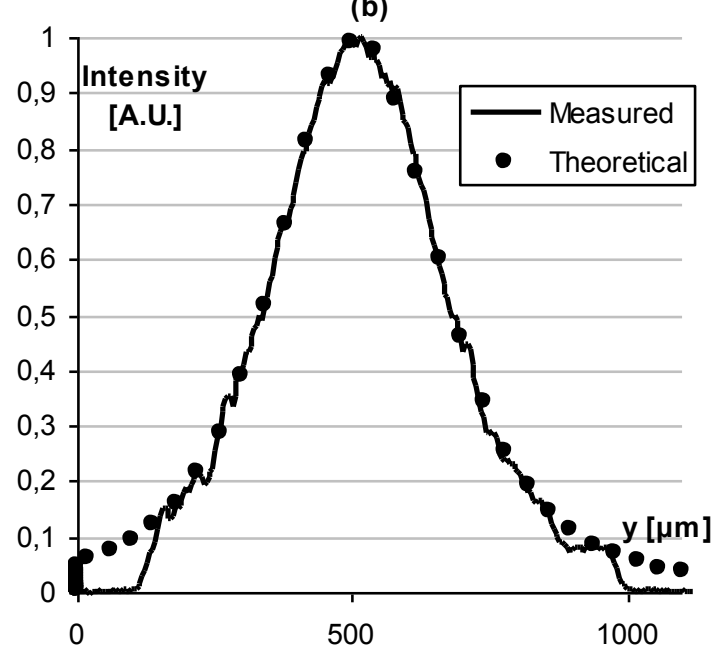

Fig. 1. The comparison of the calculated model (theoretical) and the measured data (experimental) at a distance of $1 \mathrm{~mm}$ behind the LD.
(a) The intensity distribution in the $x$ direction
(b) The intensity distribution in the $y$ direction

The overall relative error of fitting $\Delta$ was estimated by comparing a surface under the measured $\left(S_{m}\right)$ and theoretical curves $\left(S_{t}\right)$ :

$$
\Delta=\frac{\left|S_{t}-S_{m}\right|}{S_{m}} \cdot 100 \% .
$$

The overall error in the observable area is $2.2 \%$ in the case of fitting the slow axis $(x)$ and $1.7 \%$ for the fast axis $(y)$.

The measurement of the light field distribution behind the LD output plane coincides exactly with the numerical simulations. Therefore, our approach leads to correct results for properly matched parameters $N, \sigma$ and $p$. The number of coherent sources $N$ and the constant $\sigma$ characterize the light filed distribution in the $x$ direction, while the parameter $p$ describes the light field distribution in the $y$ direction. Each of $N$ sources is treated as a coherent one. Then, the incoherent summation of $N$ fields results in the output intensity distribution.

The above presented model can be used to design diffractive structures that collimate or focus light. The example of a phase diffractive structure focusing the light is presented below. The structure is designed both to compensate the phase distribution and to focus the light into a small area. Then one can use, for example a pinhole, to get a well collimated or focused beam.

At first we should design a corrective element that compensates the phase distribution of the light emitted by the LD. We can't determine the phase distribution of light coming from mutually incoherent sources. Therefore we calculated the conjugated phase of the non-paraxial field emitted by a single coherent source at a given distance from the LD (here $1 \mathrm{~mm}$ ). Thus we obtained the phase compensation only for a small region of the LD's active area. Nevertheless we assume that such compensation is approximately accurate for the adjacent regions. Therefore the aperture of the corrective element is divided into some parts as it is demonstrated in Fig. 2. Each part compensates strictly the phase coming from a coherent elementary source situated in front of the part. The assumption that the neighboring regions produce similar phase distribution is a crucial point of our model but as we will demonstrate in the experiment, it leads to a satisfactory light concentration.

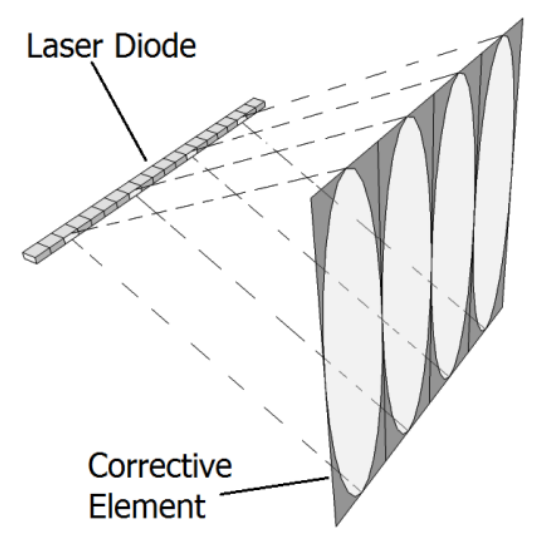

Fig. 2. The example of the corrective element consisting of four parts.

The focusing structure combined with the corrective element defines the final phase distribution of the diffractive element.

We designed an element focusing light according to the setup presented in Fig. 3. 


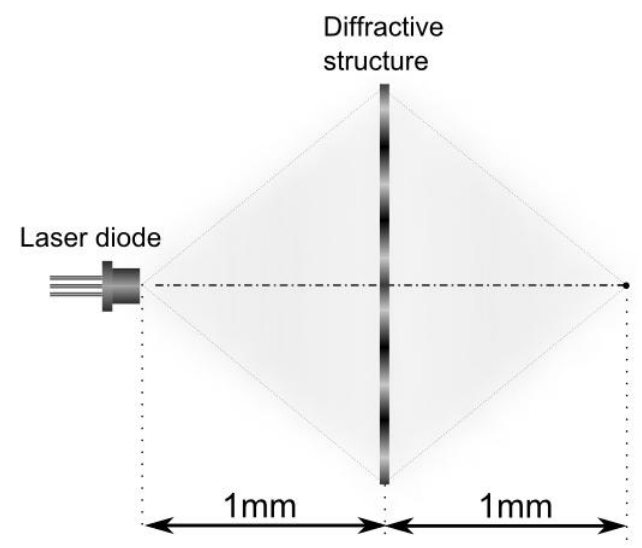

Fig. 3. Light focusing element distant $1 \mathrm{~mm}$ from LD and the output plane.

The corrective part of the structure consists of 6 parts having a width of $37.5 \mu \mathrm{m}$. They define the whole aperture of the element in the form of a rectangle with the dimensions $225 \mu \mathrm{m} \times 540 \mu \mathrm{m}$. At least $90 \%$ of the energy of the light emitted by the LD is directed onto the aperture.

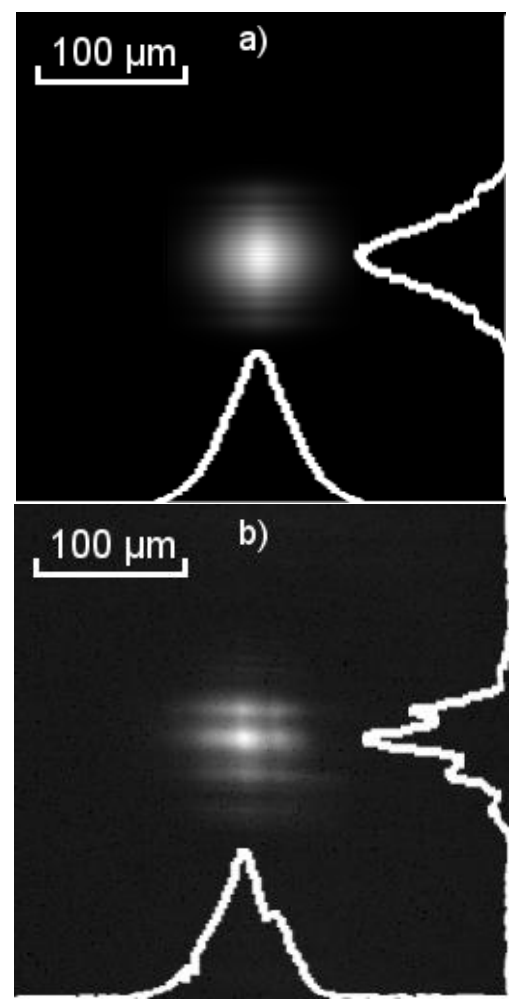

Fig. 4. Intensity distributions of focused light: (a) numerical simulation; (b) experimental verification.

As the focusing part of the element, an elliptical lens was chosen. The mentioned rectangular aperture causes asymmetrical diffraction in perpendicular directions. The elliptical lens compensates this effect forming a symmetrical focal spot [15].

Figure 4 presents the numerical simulation and experimental verification of light concentration. The diffractive element was fabricated by laser lithography on a glass substrate. The numerical simulation enabled the calculation of the diffraction efficiency of the manufactured structure. The efficiency within a circle of $50 \mu \mathrm{m}$ diameter was $44 \%$. Generally, the simulation coincides with the experiment. The intensity oscillations visible in Fig. 4b are probably caused by fabrication errors.

A numerical model of the high power laser junction was proposed. It was shown that the numerical simulations correspond precisely to the measured experimental data. The presented method of design can be applied to focusing or collimating light emitted by edge lasers.

It is worth mentioning that light is transformed by only one diffractive structure, which facilitates the manufacturing of an LD together with a focusing element. The presented approach can be expanded to "long" multiple junction lasers.

We thank Dr. Agnieszka Siemion for a valuable discussion and technical help, the Polish Holographic Systems for sharing KineMax technology and Orteh sp. $\mathrm{z}$ o.o. for sharing the software.

\section{References}

[1] E. Wolf, J. Opt. Soc. Am. 72, 343 (1982)

[2] E. Wolf, J. Opt. Soc. Am. A 1, 541 (1984).

[3] A. Naqwi and F. Durst, Appl. Opt. 29, 1780 (1990).

[4] J. Yang, T. Chen, G. Ding and X. Yuan, Proc. SPIE 6824, 68240A (2007).

[5] X. Zeng, A. Naqwi, Appl. Opt. 32, 4491 (1993)

[6] Y. Li and J. Katz, Appl. Opt. 35, 1442 (1996).

[7] X. Zeng, Z. Feng, Y. An, Appl. Opt. 43, 5168 (2004)

[8] J. Terv1o, J. Turunen, P. Vahimaa, and F. Wyrowski, J. Opt. Soc. Am. A 27, 2004 (2010)

[9] J. Turunen, J. Mod. Opt. 58, 509 (2011).

[10] H. Partanen, J. Tervo, and J. Turunen, Appl. Opt. 52, 3221 (2013).

[11] M. Sypek, Opt. Commun. 116, 43 (1995).

[12] Z. Jaroszewicz, A. Kołodziejczyk, M. Sypek, C. Gomez-Reino, J. Mod. Opt. 43, 617 (1996).

[13] M. Sypek, C. Prokopowicz, M. Górecki, Opt. Eng. 42, 3158 (2003).

[14] C. Prokopowicz, Proc. SPIE 5484, 482 (2004).

[15] A. Sobczyk, Z. Jaroszewicz, A. Kolodziejczyk, A. Kowalik, Cz. Prokopowicz, M. Sypek, J. Opt. 15, 025702 (1-5) (2013). 